\title{
Erratum: Shadow and quasinormal modes of a rotating loop quantum black hole [Phys. Rev. D 101, 084001 (2020)]
}

Cheng Liu, Tao Zhu๑, Qiang Wu, Kimet Jusufi, Mubasher Jamil, Mustapha Azreg-Aïnou, and Anzhong Wang

(2) (Received 19 March 2021; published 14 April 2021)

DOI: $10.1103 /$ PhysRevD.103.089902

In the published version of this paper, the expression $R(r)$ in Eq. (34) should be replaced by,

$$
R(r)=[X(r) E-a L]^{2}-\Delta(r)\left[\mathcal{K}+(L-a E)^{2}\right],
$$

where $\Delta(r)$ is defined by Eq. (18), but now $X(r) \equiv k+a^{2}$, instead of $r^{2}+a^{2}$. Then, Eq. (42) should be replaced by

$$
2 g(r) h(r) \frac{d X(r)}{d r}-X(r) \frac{d}{d r}[g(r) h(r)]=0,
$$

which determines the radius of the photon sphere, $r_{\mathrm{ps}}$. Since the numerator of the above equation is a polynomial of order 7 in terms of $r$, we cannot solve it explicitly to provide an analytical solution. As a result, Eq. (43) does not hold any longer, while Eq. (44) should be replaced by

$$
\xi^{2}+\eta=\frac{h\left(r_{\mathrm{ps}}\right)}{f\left(r_{\mathrm{ps}}\right)},
$$

and Eq. (53) by

$$
R_{s}=\sqrt{\alpha^{2}+\beta^{2}}=\frac{h\left(r_{\mathrm{ps}}\right)}{f\left(r_{\mathrm{ps}}\right)} .
$$

Since now $r_{\mathrm{ps}}$ cannot be given explicitly, Eqs. (55) and (56) also need to make the corresponding corrections. However, when $r_{\mathrm{ps}}$ is sufficiently large, they still represent very good approximations.

On the other hand, for the rotating case, if we plug the relations $r_{+}=2 M /(1+P)^{2}, r_{-}=2 M P^{2} /(1+P)^{2}$ and $r_{*}=2 M P /(1+P)^{2}$ into the functions $f(r), g(r), h(r), X(r)$ and $\Delta(r)$, we find $X=X(r, M, a, P)$ and $\Delta=\Delta(r, M, a, P)$. Then, Eq. (45) should be replaced by,

$$
\xi=\frac{X_{\mathrm{ps}} \Delta_{\mathrm{ps}}^{\prime}-2 \Delta_{\mathrm{ps}} X_{\mathrm{ps}}^{\prime}}{a \Delta_{\mathrm{ps}}^{\prime}}, \eta=\frac{4 a^{2} X_{\mathrm{ps}}^{\prime 2} \Delta_{\mathrm{ps}}-\left[\left(X_{\mathrm{ps}}-a^{2}\right) \Delta_{\mathrm{ps}}^{\prime}-2 X_{\mathrm{ps}}^{\prime} \Delta_{\mathrm{ps}}\right]^{2}}{a^{2} \Delta_{\mathrm{ps}}^{\prime 2}}
$$

where the right-hand sides are evaluated at $r=r_{\mathrm{ps}}$, and the prime denotes derivative with respect to $r$. Hence, Eq. (46) should be replaced by

$$
X(r, M, a, P)=a^{2}+\frac{r^{4}+a_{0}}{(2 M P+r)^{2}}, \Delta(r, M, a, P)=a^{2}+\frac{r^{2}(2 M-r)\left(2 M P^{2}-r\right)}{(2 M P+r)^{2}},
$$

while Eq. (52) now reads,

$$
\alpha^{2}+\beta^{2}=\frac{4 X^{\prime} \Delta\left(X^{\prime}-\Delta^{\prime}\right)}{\Delta^{\prime 2}}+2 X-a^{2}
$$


The above typos are also present in our numerical codes, but we have verified that the corrections to the shadow of the loop quantum black hole are small, and only slightly changed the size and shape of the shadow, so no corrections are needed in the corresponding figures.

In addition, the relative size of the shadow remains the same, and our main conclusions still hold. This is mainly because the function $k=\left(r^{4}+a_{0}^{2}\right) /\left(r+r_{*}\right)^{2}$ given in Eq. (20) approaches $r^{2}$ for sufficiently large $r$, so all the conclusions and results obtained in this paper remain valid in the limit of large $r$.

We sincerely apologize to readers for any inconvenience caused by these typos, and for more details, please see the revised version of our paper in arXiv:2003.00477. 